\title{
Controlling Dynamic Stability and Active Compliance to Improve Quadrupedal Walking
}

\author{
E. Garcia and P. Gonzalez de Santos \\ Industrial Automation Institute (CSIC) 28500 Madrid, Spain \\ egarcia@iai.csic.es
}

Summary. It is widespread the idea that animal legged locomotion improves wheeled locomotion on very rough terrain. However, the use of legs as locomotion system for vehicles and robots is still far away from competing with wheels and trucks even on natural ground. Walking robots feature two main disadvantages. One is the lack of reacting capabilities from external disturbances, and the other is the very slow walking motion. Both obstacles prevent walking mechanisms from being introduced in industrial processes and from being part of service and assistance robotics. This paper is aimed at solving the two above obstacles by combining a dynamic stability margin that quantifies the impact energy that a robot can withstand, and either controlling a dynamic walk by means of active compliance, which helps the robot react to disturbances. Experiments performed on the SILO4 quadruped robot show a relevant improvement on the walking gait.

\section{Introduction}

In spite of the well known theoretical advantages of walking robots over wheeled robots to move on very irregular terrain, legged systems are still far from being considered as a locomotion system in industrial and service applications. The main obstacle found, apart from mechanical complexity, is the very low velocity that walking robots feature to alow a stable motion. Moreover, walking robots easily tumble down due to unexpected external and internal disturbances and they rarely recover from the fall. To solve this inconvenience, there is a need to generate walking gaits that react to robot dynamics at the same time that they negotiate uneven terrain. Based on biological studies, a few roboticists have attempted to solve the problem of dynamic walking in legged robots using neural oscillators. Nevertheless, very few have succeeded in implementing dynamic walking in real robots [1-4] and less have validated their results on various irregular terrains $[5,6]$.

In this paper we propose a completely different approach. Instead of using biological inspiration, we asume that nature is complicated to imitate successfully and we work on the basis of a more classical approach. Let us consider a 
quadruped walking using a predefined two-phase discontinuous gait [7] on irregular terrain. Our goal is to adaptively modify the gait to improve machine speed and stability by means of a) using the Normalized Dynamic Energy Stability Margin, NDESM [8], to control the gait that quantifies the impact energy that a robot can withstand and b) using active compliance to react to internal and external disturbances.

The use of the NDESM allows to observe how close the robot is from instability even if external disturbances occur. To counteract the measured disturbances, an active compliance approach helps reacting to them. The outline of this paper is as follows: Section 2 revises the NDESM and shows how it helps monitoring dynamic disturbances to improve stability. Then, Section 3 presents the active-compliance approach that enables improving the robot speed by maintaining null moments at the robots center of gravity (COG). The complete approach is validated through experimentation using a real quadruped robot in Section 4.

\section{Controlling Dynamic Stability}

To achieve a dynamic walk, a dynamic stability margin has to be used for controlling the gait. In this work a two-phase discontinuous gait is used for the quadruped robot. The robot walks on rough, natural ground, and it uses force sensors at the feet for ground detection. These force sensors also allow detecting obstacles in the transfer trajectories of the legs. The force sensed at the feet is used in each control cycle to compute the dynamic stability margin. In this work the NDESM has been chosen because it measures the amount of impact energy a robot can withstand without tumbling down due to dynamic effects. This stability measurement is very suitable for reactively counteract those dynamic perturbations.

The general expression of the NDESM is:

$$
S_{N D E S M}=\frac{\min \left(E_{i}\right)}{m g}, i=1 \ldots 4
$$

where $E_{i}$ stands for the stability level of the $i$ th side of the support polygon, which physically means the increment of mechanical energy required to tumble the robot around the $i$ th side of the support polygon, computed from (see Figure 1):

$$
E_{i}=m g|\mathbf{R}|(\cos \phi-\cos \varphi) \cos \Psi+\left(\mathbf{F}_{\mathbf{R I}} \cdot \mathbf{t}\right)|\mathbf{R}| \theta+\left(\mathbf{M}_{\mathbf{R}} \cdot \mathbf{e}_{\mathbf{i}}\right) \theta-\frac{1}{2} I_{i} \omega_{i}^{2}
$$

where $\mathbf{R}$ is a vector orthogonal to the $i$ th side of the support polygon that points to the COG position, $\mathbf{F}_{\mathbf{R I}}$ is the resultant inertial force acting on the COG, $\mathbf{M}_{\mathbf{R}}$ is the resultant moment acting on the COG, $I_{i}$ is the moment of inertia around the rotation axis $i, \omega_{i}$ is the initial angular velocity of the 


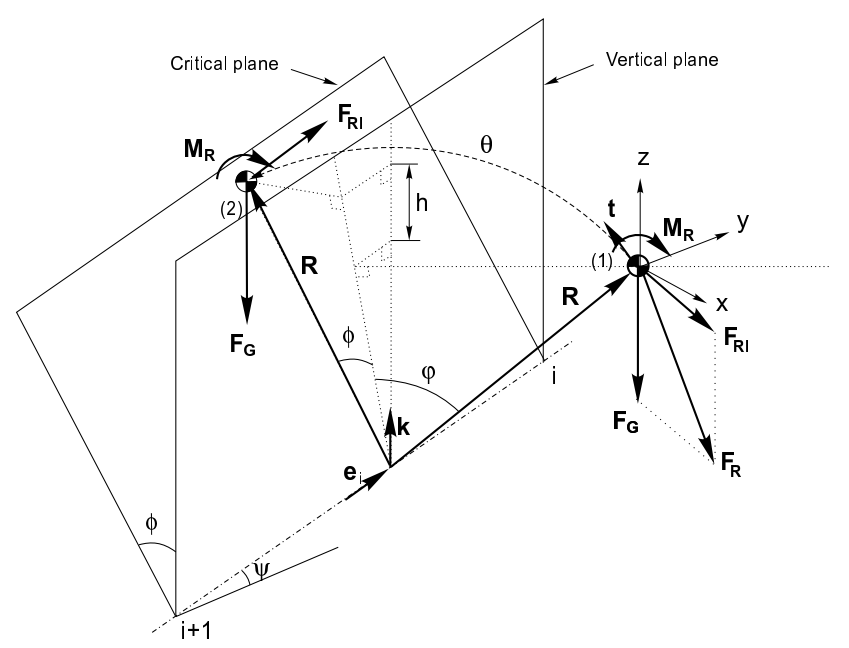

Fig. 1. Geometric outline for the computation of $S_{N D E S M}$.

COG, $\Psi$ is the terrain inclination angle, and $\phi, \varphi$ and $\theta$ are the rotation angles around the $i$ axis. $\varphi$ is the rotation angle required to position the COG inside the vertical plane (see Figure 1); $\phi$ is the angle that the COG rotates from the vertical plane to the critical plane, where the resultant moment acting on the COG vanishes. Finally, $\theta$ is the addition of both rotations. Unitary vector $\mathbf{t}$ is tangent to the COG trajectory and $\mathbf{e}_{\mathbf{i}}$ is the unitary vector that goes around the support polygon in the clockwise sense. The first three terms on the right side of equation 2 represent the potential energy required for the tumble caused by gravitational and non-gravitational forces and moments, while the fourth term represents kinetic energy (see [8] for a more detailed explanation).

Figure 2 compares the NDESM and NESM along half gait cycle of the SILO4 quadruped robot being affected by leg dynamics and manipulation dynamics while walking on a $10^{\circ}$-inclined ground. The NESM is an energybased static stability margin [9], and as the figure shows, it does not reflect the dynamics perturbing robot stability such as inertial effects at leg lift and body propulsion, vibrations due to joint elasticity at leg lift, foot placement and body support, and manipulation effects caused by a constant load of $20 \mathrm{~N}$ opposing robot motion. The NESM only reflects the effect of inclined ground, that increases the stability margin during the transference of the uphill leg and decreases it during the transference of the downhill leg. However, the NDESM presents variations during foot lift, foot placement, leg transference and body motion caused by robot inertia and joint elasticity. 


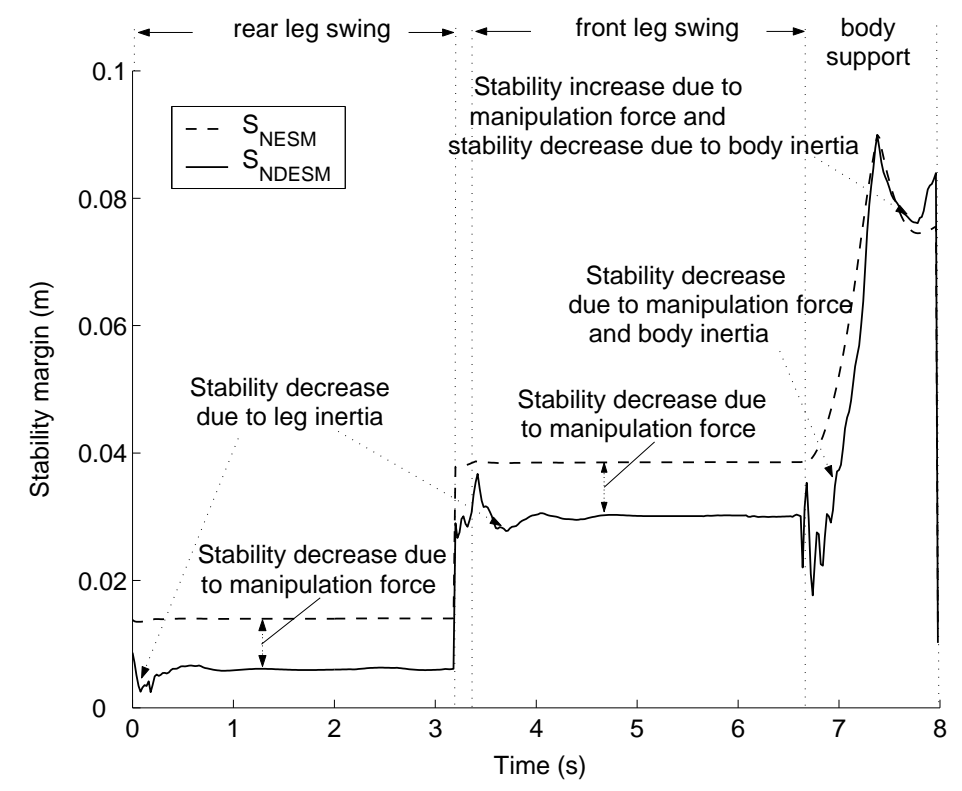

Fig. 2. NDESM vs. NESM during half gait cycle of the SILO4 quadruped robot on a $10^{\circ}$-inclined terrain when inertial, manipulation and elastic effects arise.

\section{Active Compliance in a Quadruped Gait}

The principle of active compliance in a walking robot consists in controlling the motion of each leg in support phase so that steady-state force errors are linearly proportional to displacements errors. Therefore, the compliant motion achieved is similar to that produced from a spring connecting foot directly to body following Hook's law. In this work, active compliance is realized through a velocity servo system. Foot-force errors are converted to foot-velocity errors and later mapped to desired joint speed, so the active compliance equation for each supporting leg has the form,

$$
\dot{\mathbf{q}}_{c}=\mathbf{J}^{-1}\left[\mathbf{K}_{\mathbf{p}}\left(\mathbf{X}_{d e s}-\mathbf{X}\right)+\mathbf{K}_{\mathbf{f}}\left(\mathbf{F}_{d e s}-\mathbf{F}\right)+\dot{\mathbf{X}}_{d e s}\right]
$$

where $\mathbf{X}_{d e s}$ and $\dot{\mathbf{X}}_{\text {des }}$ are vectors of desired cartesian foot position and velocity, determined by gait generation, and $\mathbf{F}_{\text {des }}$ in the vector of desired cartesian foot forces, obtained from the force-distribution algorithm. $\mathbf{X}$ and $\mathbf{F}$ are measured cartesian foot positions and forces. $\mathbf{J}$ is the jacobian matrix that transforms cartesian foot speed to joint speed. $\mathbf{K}_{\mathbf{p}}$ and $\mathbf{K}_{\mathbf{f}}$ are diagonal matrix of gains, being $\mathbf{C}_{\mathbf{R}}=\mathbf{K}_{\mathbf{f}} \cdot \mathbf{K}_{\mathbf{p}}$ the compliance ratio and $\mathbf{K}_{\mathbf{f}}{ }^{-1}$ the damping parameter. It is desired to have a big damping for the stability of the control law. Figure 3 is a block diagram of the active-compliance controller. 


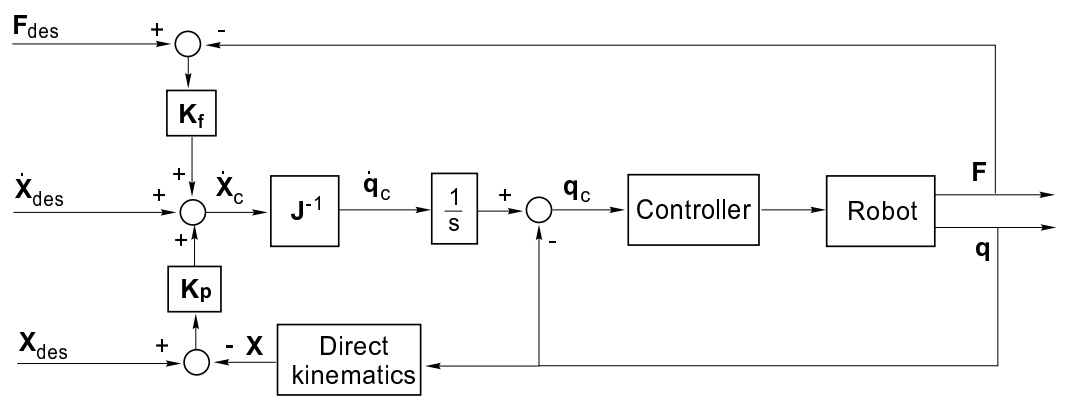

Fig. 3. Block diagram of the active-compliance controller.

The active compliance controller is used in this work to enable the robot to react to internal and external disturbances. To achieve this, the robot's COG is desired to maintain with zero moments $\mathbf{M}_{\mathbf{x}}$ and $\mathbf{M}_{\mathbf{y}}$. Also, for the system to maintain stability, the sum of vertical foot forces has to equal the resultant vertical force $\mathbf{F}_{\mathbf{z}}$ acting on the robot's COG. The problem is slightly different depending on the number of legs in support phase. During a twophase discontinuous gait, periods of three legs in support and periods of four legs in support alternate. Let $\mathbf{W}_{\mathbf{d}}$ be the vector of vertical force and pitch and roll moments desired at the robot $\mathrm{COG}$, that is:

$$
\mathbf{W}_{\mathbf{d}}=\left[\begin{array}{lll}
F_{z} & M_{x} & M_{y}
\end{array}\right]^{T}
$$

Assuming $F_{z}=m g, M_{x}=0$ and $M_{y}=0$ the resultant forces and moments at the COG have the form:

$$
\mathbf{W}_{\mathbf{d}}=\left[\begin{array}{lll}
m g & 0 & 0
\end{array}\right]^{T}
$$

where $\mathrm{m}$ is the total mass of the robot. Let also $\mathbf{F}_{\text {des }}$ be the vector of vertical foot forces desired at the feet:

$$
\mathbf{F}_{d e s}=\left[\begin{array}{llll}
F_{d e s_{1}} & F_{d e s_{2}} & F_{d e s_{3}} & F_{d e s_{4}}
\end{array}\right]^{T}
$$

During the periods of four supporting legs the equilibrium equation has the form,

$$
\mathbf{W}_{\mathbf{d}}=\mathbf{A} \mathbf{F}_{\text {des }}
$$

where $\mathbf{A}$ is a $3 \times 4$ matrix of the form:

$$
\mathbf{A}=\left\{a_{i j}\right\}
$$

with

$$
a_{1 j}=1, a_{2 j}=y_{j}, a_{3 j}=-x_{j}, j=1 . .4
$$

where $x_{j}$ and $y_{j}$ are position coordinates of foot $j$ in the body's cartesian reference frame. Note that the solution to $(7)$ is indeterminate. To solve the 


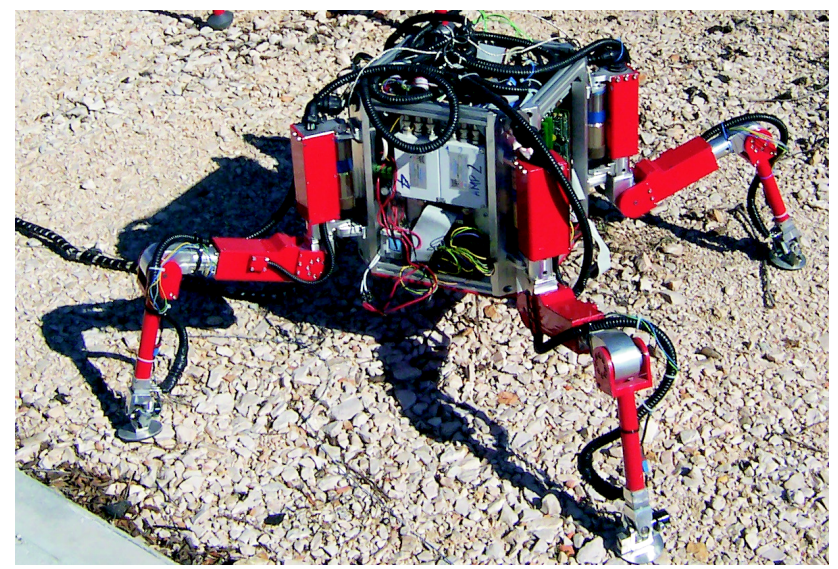

Fig. 4. The SILO4 quadruped robot

force distribution problem the indeterminacy could be eliminated by adding an optimization condition. This condition is the one that satisfies:

$$
\sum_{i \in I}\left(F_{\text {des }_{i}}\right)^{2} \rightarrow \min
$$

which has the sense of energy optimization for supporting the weight. This is solved in the following manner:

$$
\mathbf{F}_{\text {des }}=\mathbf{A}^{+} \mathbf{W}_{\mathbf{d}}
$$

where $\mathbf{A}^{+}$is the pseudoinverse of matrix $\mathbf{A}$.

During the periods of three supporting feet, matrix $\mathbf{A}$ is square $3 \times 3$, and therefore $\mathbf{A}^{+}=\mathbf{A}^{-1}$.

Once the desired foot forces that regulate the body are computed, the desired joint trajectories are modified through (3).

\section{Experimental Results}

The SILO4 quadruped robot (see Figure 4) has been used to show the improvement on dynamic walking achieved using the herein proposed approach. In this section some experiments using the SILO4 robot will be shown to report the improved functionality.

\section{Acknowledgements}

This work has been partially funded by CICYT (Spain) through Grant DPI2004-05824. The first author is supported by a postdoctoral CSIC-I3P contract granted by the European Social Fund. 


\section{References}

1. H. Kimura, S. Akiyama, and K. Sakurama, "Realization of dynamic walking and running of the quadruped using neural oscillator," Autonomous Robots, vol. 7, no. 3, pp. 247-258, 1999.

2. W. Ilg, J. Albiez, H. Jedele, K. Berns, and R. Dillmann, "Adaptive periodic movement control for the four legged walking machine bisam," in Proc. IEEE Int. Conf. Robotics and Automation, 1999, pp. 2354-2359.

3. K. Tsujita, K. Tsuchiya and Onat, "Adaptive gait pattern control of a quadruped locomotion robot," in Proc. IEEE/RSJ Int. Conf. on Intelligent Robots and Systems, 2001, pp. 2318-2325.

4. M.A. Lewis, Etienne-Cummings, M.J. Hartmann, Z.R. Xu, and A.H. Cohen, "An in silico central pattern generator: silicon oscillator, coupling, entrainment, and physical computation," Biological Cybernetics, vol. 88, pp. 137-151, 2003.

5. Y. Fukuoka, H. Kimura, and A.H. Cohen, "Adaptive dynamic walking of a quadruped robot on irregular terrain based on biological concepts," The International Journal of Robotic Research, vol. 22, no. 3-4, pp. 187-202, 2003.

6. H. Kimura and Y. Fukuoka, "Biologically inspired adaptive dynamic walking in outdoor environment using a self-contained quadruped robot: 'tekken2'," in Proc. IEEE/RSJ Int. Conf. on Intelligent Robots and Systems, 2004.

7. P. Gonzalez de Santos and M.A. Jimenez, "Generation of discontinuous gaits for quadruped walking machines," Journal of Robotic Systems, vol. 12, no. 9, pp. 599-611, 1995.

8. E. Garcia and P. Gonzalez de Santos, "An improved energy stability margin for walking machines subject to dynamic effects," Robotica, vol. 23, no. 1, pp. 13-20, 2005.

9. S. Hirose, H. Tsukagoshi, and K. Yoneda, "Normalized energy stability margin: Generalized stability criterion for walking vehicles," in Proc. Int. Conf. Climbing and Walking Robots, November 1998, pp. 71-76, Brussels, Belgium. 